\title{
Study of urban spatial patterns from SPOT panchromatic imagery using textural analysis
}

\author{
Q. ZHANG†, J. WANG H†* $^{*}$ P. GONG§ and P. SHI \\ $\dagger$ Department of Geography, The University of Western Ontario, London, \\ ON, N6A 5C2, Canada \\ $\$$ Key Laboratory of Environmental Change and Natural Disaster, the \\ Ministry of Education of China, Beijing Normal University, Beijing, 100875, \\ China \\ $\S$ Department of Environmental Science, Policy and Management, University \\ of California, Berkley, CA 94720, USA
}

(Received 9 July 2001; in final form 17 September 2002)

\begin{abstract}
The long-time historical evolution and recent rapid development of Beijing, China, present before us a unique urban structure. A 10-metre spatial resolution SPOT panchromatic image of Beijing has been studied to capture the spatial patterns of the city. Supervised image classifications were performed using statistical and structural texture features produced from the image. Textural features, including eight texture features from the Grey-Level Cooccurrence Matrix (GLCM) method; a computationally efficient texture feature, the Number of Different Grey-levels (NDG); and a structural texture feature, Edge Density (ED), were evaluated. It was found that generally single texture features performed poorly. Classification accuracy increased with increasing number of texture features until three or four texture features were combined. The more texture features in the combination, the smaller difference between different combinations. The results also show that a lower number of texture features were needed for more homogeneous areas. NDG and ED combined with GLCM texture features produced similar results as the same number of GLCM texture features. Two classification schemes were adopted, stratified classification and non-stratified classification. The best stratified classification result was better than the best non-stratified classification result.
\end{abstract}

\section{Introduction}

Beijing, the capital city of China, is currently one of the world's most populated and rapidly developing cities. At the same time, Beijing was originally planned and built as an ancient Chinese imperial capital. There are different stages in the course of development (Gaubatz 1995). Associated with the development stages, there are morphologically different built-up areas. Before 1949, Beijing was planned and built based on the Chinese ideal of a capital city (Wu 1986). The street layout of the old city was established in the 13-14th centuries and has not changed much since that

\footnotetext{
*Author for correspondence at Department of Geography, The University of Western Ontario, London, Ontario, N6A 5C2, Canada; e-mail: jfwang@uwo.ca

International Journal of Remote Sensing

ISSN 0143-1161 print/ISSN: 1366-5901 online (C) 2003 Taylor \& Francis Ltd

http://www.tandf.co.uk/journals

DOI: $10.1080 / 0143116031000070445$
} 
time. Inside the old city, there are century-old Chinese style courtyard houses. After 1949, Soviet planning ideas influenced the development of Beijing (Gaubatz 1995, Wu 1999). Many 3-5 storey workplace compounds were built after the socialist idea that individual life is organized around the workplace. After the reform and opendoor policy in 1979, the development of Beijing entered into a new era, partly influenced by western planning ideas (Gaubatz 1995). New development includes suburban 'special development zones', new medium to high-rise residential and/or office buildings, in recently built and rebuilt areas.

After the rapid development in the recent decades, Beijing has become more and more like its western counterparts in appearance. There have arisen heated conflicts between historical heritage preservation and modern development (Wu 1999). Other social-economic factors, such as the beginning of population suburbanization observed by Wang and Zhou (1999), are also expected to change the physical structure of the city. The impact of recent development of the city on the overall built environment is yet to be seen. When it comes to new development in the suburbs or redevelopment of the old city, it is important to investigate what kind of development exists around the neighbourhood, in order to assess the environmental impact, fitting-in, cost, etc, of new development or redevelopment.

Field survey and visual interpretation of aerial photographs have long been adopted as the most popular methods to generate useful information about an urban area. These methods are time-consuming and expensive. It is also difficult to keep up with the rapid development pace in big cities using traditional methods. Satellite remote sensing has the advantages of frequent revisit, global coverage and lower cost. Landsat Thermatic Mapper (TM) and SPOT multi-spectral imagery have been used for urban land cover/land use mapping (Harris and Ventura 1995, Mesev 1998). Compared with aerial photography, satellite imagery has not received as much focus in urban applications, mainly because the spatial resolution of the earlier generations of space-borne sensors is limited (Welch 1982, Forster 1985). However, this situation has changed, with the launch of several medium to very high spatial resolution satellites. The SPOT satellite collects panchromatic data at a ground pixel resolution of $10 \mathrm{~m}$. The new IKONOS satellite is capable of collecting multi-spectral data of $4 \mathrm{~m}$ spatial resolution and panchromatic data of $1 \mathrm{~m}$ spatial resolution. This increasing spatial resolution of satellite sensor data is approaching and fulfilling the spatial resolution requirement of urban applications (Zhang and Wang 2003).

The data processing method is another issue that limits the utility of higher spatial resolution remote sensing imagery. Automated image interpretation has been developed and used for land cover/land use mapping from medium-low spatial resolution multi-spectral remotely sensed data. These automated classifiers assume that different surface materials have different spectral characteristics, so that the grey-level digital values recorded by sensors are sufficient indicators to classify these pixels into different classes. With the increase of spatial resolution, between-class spectral confusion and within-class spectral variation were found to increase for land cover/land use studies (Haack et al. 1987, Barnsley and Barr 1996). Spectral information alone is not enough to map urban land cover/land use from mediumhigh spatial resolution remotely sensed data. Furthermore, many satellite sensors operate at a higher spatial resolution in panchromatic channels than multi-spectral 
channels, such as SPOT, Landsat 7, IRS and most recently, IKONOS. Though the panchromatic channels of these sensors provide more spatial detail than their multi-spectral channels, automated multi-spectral classification is not appropriate for analysis of panchromatic imagery.

Texture and structure analyses are among the approaches used to incorporate spatial information. Textural information has been an important factor in visual image interpretation. It takes into consideration the distribution and variation of neighbourhood pixel values. Haralick et al. (1973) developed a set of texture statistics for image classification based on Grey Level Co-occurrence Matrix (GLCM). Studies by Marceau et al. (1990) and Gong et al. (1992) demonstrated that these textural statistics were useful to resolve spectral confusion between land cover classes. Structural information, such as road density (Wang and Howarth, 1991, Wang 1995, Zhang et al. 2002) and edge density (Gong and Howarth 1990), has also proven to be helpful for urban area segmentation and land cover classification.

The focus of this study is to evaluate the capability of using textural analysis on $10 \mathrm{~m}$ spatial resolution SPOT panchromatic (PAN) imagery for mapping the unique urban spatial patterns of Beijing. SPOT panchromatic imagery provides more spatial details than widely used $30 \mathrm{~m}$ spatial resolution Landsat TM or $20 \mathrm{~m}$ spatial resolution SPOT multi-spectral imagery. Many low-rise, multi-storey and high-rise buildings in the study area can be identified visually from the SPOT panchromatic image by their size, shape and arrangement. The main objective of this study is to evaluate the performance of different texture features and their combinations for the mapping of urban spatial patterns using SPOT panchromatic images.

The significance of this study is due to (1) the use of SPOT panchromatic imagery, which has a higher spatial resolution than the widely used Landsat TM or SPOT multi-spectral imagery, but has been the subject of fewer studies; and (2) the research being on a developing Chinese city, which has a denser built-up area and shows much higher spatial variation than the more commonly studied North American cities.

The SPOT panchromatic imagery used in this study was collected on 28 August 1994. As shown in figure 1 , a sub-scene $(1024 \times 1024$ pixels $)$ was selected for this study, which is located in the northeast quadrant of the city. At the urban-rural fringe of Beijing, there were new high-density residential areas as shown at ' $F$ ' and around ' $J$ ' in figure 1. Inside the Second Ring Road (indicated by '2' in figure 1), the site of the ancient city wall, was the old city with lower housing density. Different parts of the city, which were built at different periods with different building styles, can be recognized in the image. The Second Ring Road is the dividing line between the century-old inner city and the external city built mainly during the last half century, especially in the last two decades. Figure 2 displays some enlarged samples of texturally different patterns found in the urban and suburban areas. Each sample in figure 2 contains $40 \times 40$ pixels.

\section{Textural measures}

Texture refers to 'the spatial distribution of tonal variations' (Haralick et al. 1973). As discussed by Haralick (1979), there are two approaches to textural description: statistical and structural. Statistical models obtain image texture 


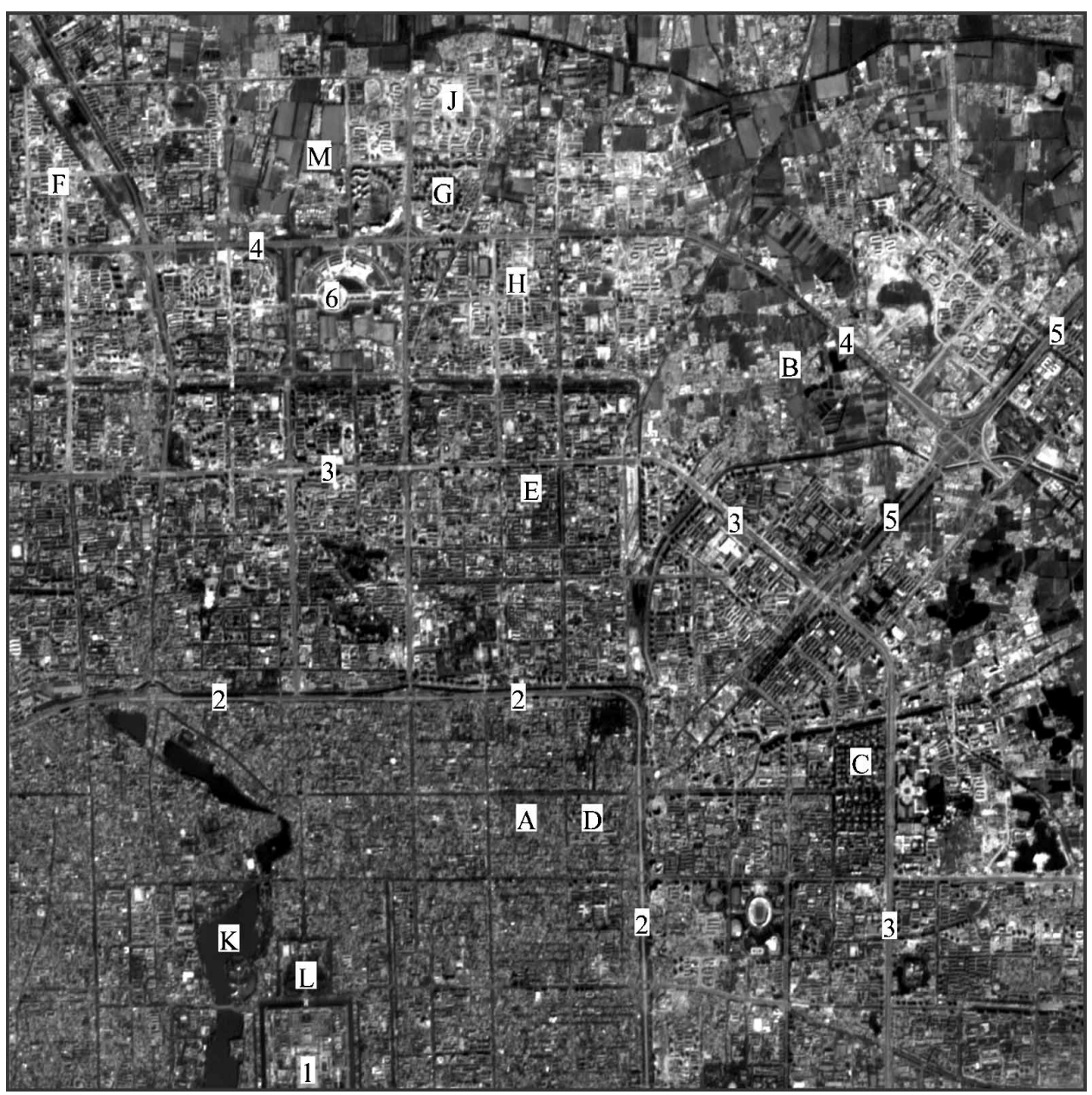

Figure 1. SPOT panchromatic image of Beijing, 28 August 1994. Numbered landmarks are: (1) The Forbidden City, (2) Second Ring Road, (3) Third Ring Road, (4) Forth Ring Road, (5) Airport Expressway, and (6) Olympic Sports Center. Enlarged image chips of the letter-labelled areas are shown in figure 2.

measurements by calculating the spatial dependency of grey-level tones, while structural models describe texture as primitives and their attributes and relationships. Statistical approaches for texture are more widely used in image analysis of urban areas because statistical textural features are easier to calculate than structural primitives, with the exception of analysis of edges as a structural approach (Webster 1996).

There are several ways to quantify texture of an image, such as the GLCM, Grey-Level Run Length Matrix (GLRLM) and Grey-Level Difference Vector (GLDV) (Haralick 1979, Weszka et al. 1976, Conners and Harlow 1980). In order to calculate texture features for each pixel in an image, a moving window is usually used to define the neighbourhood of a pixel, and the texture measurement 


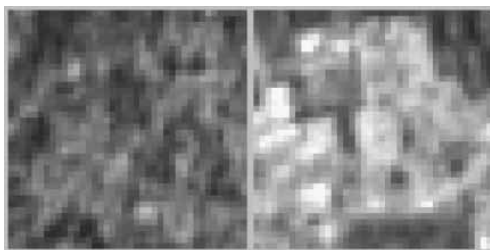

(A)

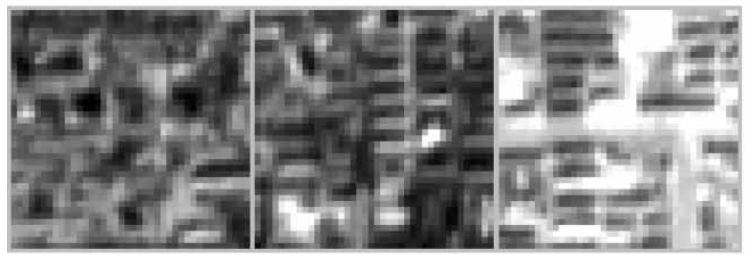

(D)

(E)

(F)

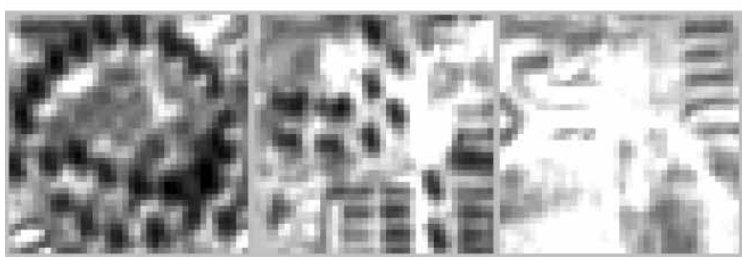

G. High-rise residential towers

H. Just-built high-rise area

J. Construction site
D. Old multi-storey area

E. New multi-storey area

F. Most recently built multi-storey residential area
(G)

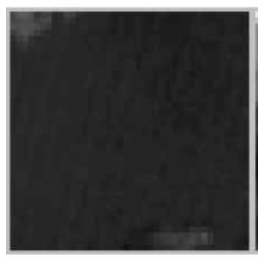

(K)
(H)

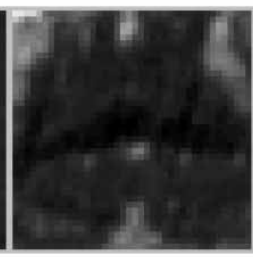

(L)

Figure 2. Enlarged samples from SPOT image showing different structures.

calculated from the window is assigned to the centre pixel. Among many methods, the GLCM method was found to be more effective (Conners and Harlow 1980). GLCM is a matrix derived from the grey-level image, which shows the jointprobability of distribution of a pair of grey levels, separated at a certain distance and a certain orientation.

Eight texture features calculated from the GLCM method are evaluated in this study, including homogeneity $(H O M)$, contrast $(C O N)$, dissimilarity $(D I S)$, mean $(M E A N)$, standard deviation $(S D)$, entropy $(E N T)$, angular second moment $(A S M)$ and correlation $(C O R)$. In some studies, homogeneity is called inverse different moment, contrast is called inertia and, angular second moment is called energy or uniformity (Baraldi and Parmiggiani 1995). The definitions of the eight GLCM 
texture features applied in this study are as follows:

$$
\begin{gathered}
H O M=\sum_{i=0}^{N-1} \sum_{j=0}^{N-1} \frac{P_{i, j}}{1+(i-j)^{2}}, \\
C O N=\sum_{i=0}^{N-1} \sum_{j=0}^{N-1} P_{i, j}(i-j)^{2}, \\
\text { DIS }=\sum_{i=0}^{N-1} \sum_{j=0}^{N-1} P_{i, j}|i-j|, \\
M E A N=\mu_{I}=\sum_{i=0}^{N-1} \sum_{j=0}^{N-1} i P_{i, j}, \\
\text { SD } \sigma_{I}=\sqrt{\sum_{i=0}^{N-1} \sum_{j=0}^{N-1} P_{i, j}\left(i-\mu_{I}\right)^{2},} \\
\text { ENT }=\sum_{i=0}^{N-1} \sum_{j=0}^{N-1}-P_{i, j} \ln P_{i, j}, \\
A S M=\sum_{i=0}^{N-1} \sum_{j=0}^{N-1} \frac{\left(i-\mu_{I}\right)\left(j-\mu_{J}\right)}{\sigma_{I} \sigma_{J}}, \\
\sum_{j=0}^{N-1},
\end{gathered}
$$

where $N$ is the number of grey levels; $P$ is the normalized symmetric GLCM of dimension $N \times N$; and $P_{i, j}$ is the $(i, j)$ th element of $P ; \quad \mu_{J}=\sum_{i=0}^{N-1} \sum_{j=0}^{N-1} j P_{i, j}$; $\sigma_{J}=\sqrt{\sum_{i=0}^{N-1} \sum_{j=0}^{N-1} P_{i, j}\left(j-\mu_{J}\right)^{2}}$.

HOM measures local homogeneity, and results in a large value if the elements of the GLCM are concentrated on the main diagonal. CON measures local spatial frequency; if the GLCM has large off-diagonal elements, the local window has high contrast. DIS is similar to $C O N$-high contrast of the local window indicates high $D I S$ value. $M E A N$ and $S D$ measure mean and standard deviation in terms of the GLCM. ENT measures disorder of the image, while $A S M$ indicates local uniformity. For details about the GLCM method refer to Haralick et al. (1973). Window size, texture statistics, quantization level, the inter-pixel distance and orientation, and the spatial resolution of the image are the factors that contribute to the performance of textural statistics from GLCM method (Marceau et al. 1990, Gong et al. 1992, Arzandeh and Wang 2002).

The GLCM method is computationally demanding. For example, for an 8-bit image, a $256 \times 256$ matrix may be constructed and then the texture features are calculated from this matrix. One matrix is constructed for one pixel and the computation is repeated for every pixel in the image. Hornstra et al. (1999) 
proposed a very simple texture measurement, called 'intra-pixel reflectance variability (IPRV)'. It measures the number of different grey levels occurring in a local window from the original imagery. We also studied this simple texture feature. For simplification, we call it NDG (Number of Different Grey-levels). For example, for a $3 \times 3$ window, the range of $\mathrm{NDG}$ is $1-9$, where ' 1 ' represents the homogeneous situation and ' 9 ' represents the opposite situation.

Besides statistical texture features, we studied edge density as a structural texture feature. In this study, edge density (ED) was produced through the following procedures, which are similar to the method used by Gong and Howarth (1990). Firstly, the SPOT image was filtered using a Laplacian high pass filter. Secondly, edges were found by thresholding the filtered image. Then, the binary edge map was coded as ' 255 ' and ' 0 ', with ' 255 ' representing edge pixels. Finally, an average filter was used to produce the edge density map.

\section{Research questions}

Since the GLCM textures statistics were the most widely used in the reported studies especially in radar image analysis (Arzandeh and Wang 2002) and were found to be better than some other methods, we chose to focus on the GLCM textures in this study. As described above, two other texture features, NDG and ED, were also evaluated with the GLCM textures. This study was carried out to answer the following questions:

1. How do single and more than one GLCM texture features perform?

2. What happens when GLCM texture features are combined with other texture features (here, NDG and ED)?

3. Do texture features perform differently in the visually textural-different old city and external city of this particular study area?

\section{Research design}

A supervised classification approach was adopted in this study. The difference with traditional multi-spectral classification is that textural channels were used for the classification instead of spectral channels. To answer the research questions, different combinations of GLCM texture features and GLCM with other texture features were studied (figure 3 ). The same classification algorithm was applied to a single GLCM texture, then combinations of two GLCM textures, combinations of three GLCM textures, and so on. The best classification results were found for each group. Then NDG and ED were studied alone and also added to the classification with the best GLCM combinations. In order to evaluate the effect of texture features, a classification of the single-channel SPOT tonal image was also produced using the same classification algorithm. This classification procedure was carried out for the whole study area, and for three textural-different regions of the study area, namely old city, external city and non built-up areas. The accuracy of final classification results was evaluated using random samples. To determine the ground status of the samples, more detailed street maps were consulted and field checking were conducted. 


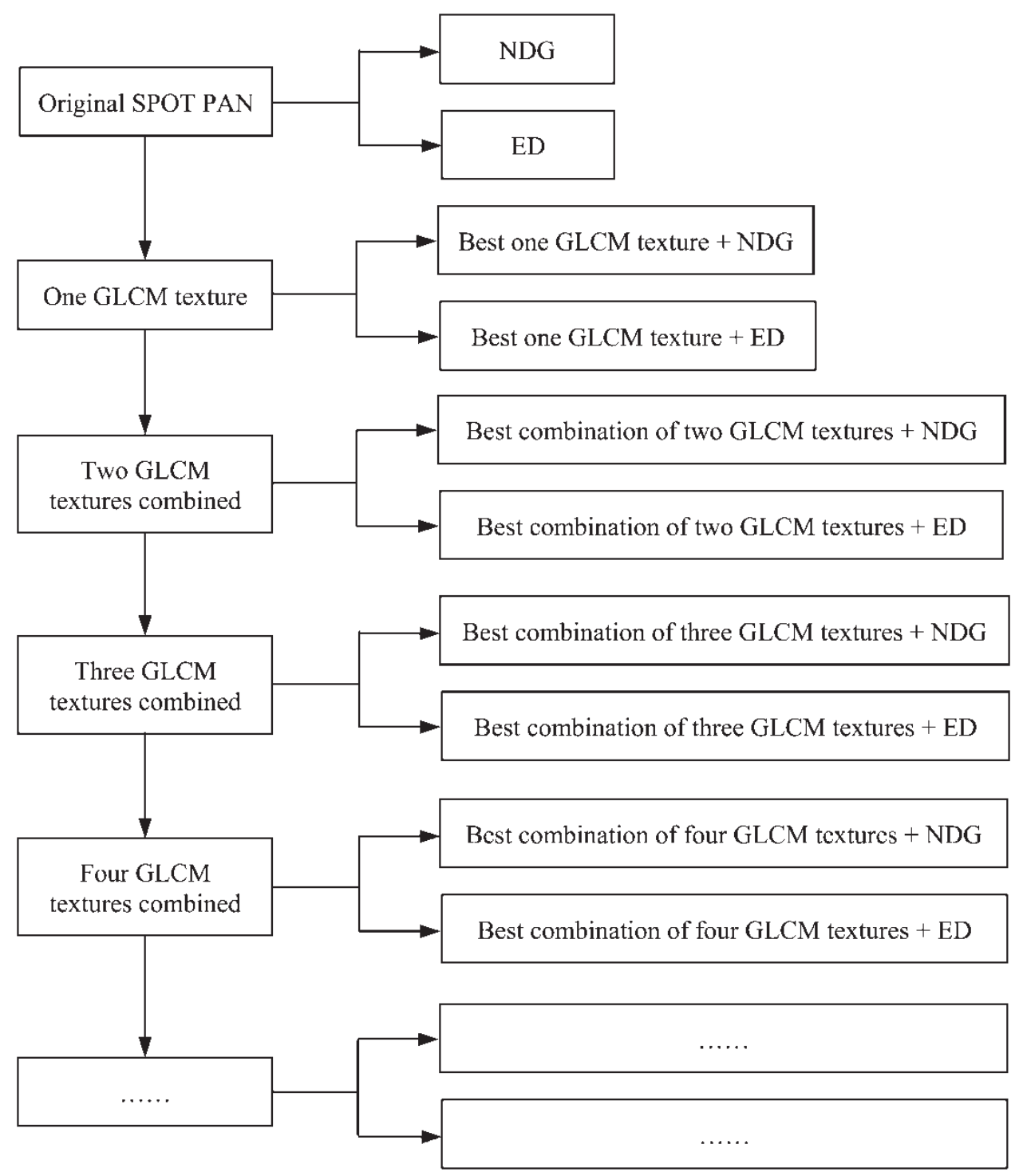

Figure 3. Flow chart of experiments on different combinations of texture features.

\subsection{Quantification of texture channels}

The main purpose of this study is to evaluate the performance of different texture features, especially GLCM texture features. Factors that influence the performance of GLCM textures such as window size, inter-pixel spatial relationship, quantization level, and the spatial resolution of the image, were kept constant. For more exhaustive experiments on these factors for textural analysis, readers are referred to Marceau et al. (1990), Shaban and Dikshit (2001), and Arzandeh (2001).

In this study, for all the GLCM texture features, the same inter-pixel distance (1 pixel) and directional invariance (4-direction average) were specified. All GLCM texture statistics were firstly calculated in 32-bit real numbers and then scaled to 
8-bit using a simple linear scaling. The NDG channels were also scaled to 8-bit range to match the quantization level of other texture channels. Different window sizes from $3 \times 3$ to $9 \times 9$ were tested and we finally selected $7 \times 7$ because this window size was found to be generally good for recognizing built-up patterns using the GLCM textures in this study. Also the border blurring problem which happens when using a large window size was not obvious at this window size.

\subsection{Stratification of the study area}

Preliminary classification of entire study area showed that there is substantial confusion between agricultural fields and some homogeneous areas in the city. There is also confusion between different built-up classes such as old multi-storey, newly-built multi-storey and high-rise areas. Our knowledge about the city tells us that the newly-built multi-storey buildings were distributed at the outskirts of the city in 1994. Visual analysis of the image (figure 1) also suggested that there are generally three regions with different spatial patterns: old city, external city and suburban agricultural areas.

To reduce the confusion and increase classification accuracy, we decided to stratify the study area into the above three regions: region 1 -old city, region 2external city and region 3-non built-up areas. By thresholding texture NDG5 (where the number 5 denotes that it was from a $5 \times 5$ window), we firstly split the study area into two regions: built-up and non built-up. Some small polygons inside these two regions were removed to avoid over-fragmentation of the regions. The threshold was selected when there were no built-up areas left inside the region 3 by checking the thresholding result against ground information and reference maps. No single texture can separate the old city and outside built-up areas, but visually, the Second Ring Road (labelled as ' 2 ' in figure 1) can be clearly seen as the dividing line. Then the old city was separated from other built-up areas by drawing the boundary manually. The stratification is shown in figure 4 .

The same classification scheme was used for all classifications, both un-stratified and stratified. This is to say that the same class has the same identifier in the classification results. Un-stratified classifications have the highest number of classes and the classes in the stratified classifications were a subset of the classes in the whole study area. Different classification sub-schemes were designed for the three stratified regions. For example, no agricultural sub-classes were allowed in region 1, and no built-up sub-classes were allowed in region 3. 'New multi-storey' sub-classes would not exist in region 1, because 'new multi-storey' refers to new apartment/ office buildings built in the 1980s and 1990s outside of the old city. The classification sub-schemes were expected to reduce confusion between classes in the final classification results.

In the training stage, a total of 20 classes were trained for the whole study area, for both un-stratified and stratified classifications. For the classification of the same region, the same training statistics were used, i.e. the same training channels were used for all un-stratified classifications, and for the stratified classification of the old city, etc.

Different combinations of texture features as shown in figure 3 were tested for classifying each of these regions. Classifications were performed for these three regions separately and the best results for each region were combined in the end to produce a complete classification result for the whole study area. 

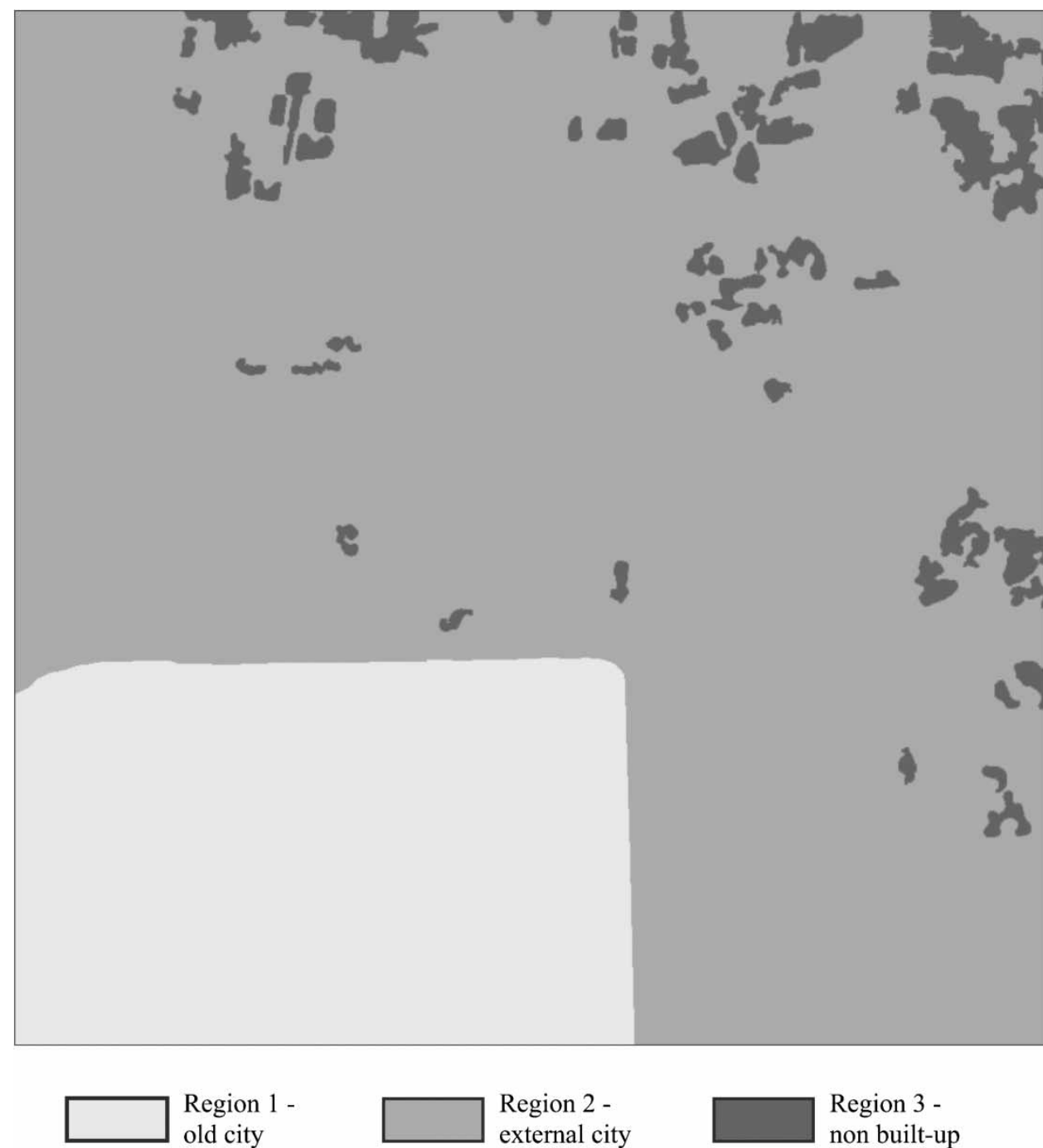

Region 2 external city

\section{Region 3 -}

non built-up

Figure 4. Stratification of the study area into three regions.

\section{Results and discussion}

The supervised classification produced a total of 20 preliminary classes for the whole study area, including the 12 categories shown in figure 2. Some classes were aggregated into one class following the classification. There are two reasons behind the aggregation. One reason is that not all the spectrally and texturally different classes correspond to information classes for the study of urban spatial patterns. For example, since agricultural areas are not our focus in this study, a few classes of 'agriculture' were aggregated into one 'agriculture' class. Another example is there is no definable timeline between 'new multi-storey area' and 'most recently built multi-storey area' in figure 2, so these two categories were aggregated into 'new multi-storey area'. The other reason for the aggregation is to reduce the confusion between the final classes. The same example of 'new' and 'most recently 
built' multi-storey areas helps explain this. Though 'most recently built' areas may appear slightly brighter than the 'new multi-storey areas', there is no practical need to distinguish them in the final results. This is also true for 'old' and 'new' low-rise areas. Final aggregated classes for all classification results include: (1) open space (water, trees and grass); (2) low-rise area (no more than three storeys); (3) old multi-storey area (pre-1979); (4) new multi-storey area (after 1979); (5) high-rise area (more than eight storeys); (6) roads; (7) construction sites; and (8) agriculture. After the aggregation, clusters of less than five pixels were filtered to reduce the 'salt and pepper' effect resulting from per-pixel classification. The accuracy assessment was performed on all aggregated classification results.

It is worthwhile to note that accuracy of the automatic classification performed on the original SPOT PAN image is poor. The overall accuracy of that classification is $27 \%$ and Kappa coefficient is 0.17 .

\subsection{Results from un-stratified classification}

\subsubsection{Performance of the GLCM texture features}

The classification accuracy for the whole study area using different numbers of the GLCM texture features is shown in table 1 . We can see that many combinations of textural channels used in classification greatly improved the result from original SPOT Panchromatic imagery, especially when three or more texture features were used. When using five GLCM texture features, we were able to achieve an overall accuracy of $71 \%$ with a Kappa coefficient of 0.66 for the whole study area.

As shown in table 1, when a single GLCM texture feature is used, there is some improvement in terms of accuracy compared with the original image. But, overall, the performance of single texture features is poor. The best result is from the texture feature DIS, but it only achieved an overall accuracy of $38 \%$. Some classes, such as roads and high-rise areas, are totally missing from the classification results of a single texture feature.

When two GLCM texture features were combined, there is a significant improvement in classification accuracy. For eight GLCM textures, there are 28 different combinations of two and the top five combinations are listed in table 1. The best combination of two textures, with $59 \%$ overall accuracy, is $M E A N+E N T$. We also noticed that the best six combinations of two are $M E A N$ combined with another texture feature. So we decided to keep $M E A N$ in the combination when we tested combinations of three or more GLCM texture features.

The top five combinations of three texture features out of a total of 21 combinations are shown in table 1. One of the three texture features is always $M E A N$. Again, the result from three textures is much better than the combinations of two textures. $M E A N+E N T+C O R$ achieved the best result with an overall accuracy of $67 \%$.

After adding one more texture feature to the combinations, slight improvement is observed (table 1). From the best combination of three texture features to the best of four texture features, the overall accuracy is only improved by $3 \%$. As seen from table 1, combinations of five texture features did not greatly improve the result either, so the experiment was stopped at combinations of five GLCM texture features.

Some basic statistics were also calculated from combinations of different 
Table 1. Classification accuracy for the whole study area using texture features.

\begin{tabular}{lcc}
\hline Input channels & Overall accuracy $(\%)$ & Kappa $\times 100$ \\
\hline Original SPOT PAN & 27.0 & 16.6 \\
One GLCM texture feature & & \\
$D I S$ & 37.8 & 27.5 \\
$H O M$ & 37.3 & 26.7 \\
$S D$ & 37.1 & 25.4 \\
$A S M$ & 35.2 & 22.6 \\
$E N T$ & 30.5 & 19.9 \\
$C O N$ & 30.2 & 20.0 \\
$M E A N$ & 28.9 & 18.9 \\
$C O R$ & 16.7 & 5.9 \\
Combinations of two GLCM texture features (top five combinations) & \\
$M E A N+E N T$ & 59.3 & 52.7 \\
$M E A N+D I S$ & 58.8 & 51.8 \\
$M E A N+H O M$ & 58.5 & 51.1 \\
$M E A N+S D$ & 56.6 & 49.6 \\
$M E A N+C O N$ & 56.0 & 48.8 \\
Combinations of three GLCM texture features (top & five combinations) & \\
$M E A N+E N T+C O R$ & 67.3 & 61.8 \\
$M E A N+D I S+C O R$ & 67.3 & 61.8 \\
$M E A N+D I S+S D$ & 66.8 & 61.0 \\
$M E A N+H O M+C O R$ & 66.6 & 60.9 \\
$M E A N+H O M+S D$ & 66.3 & 60.5 \\
Combinations of four GLCM texture features (top five combinations) & \\
$M E A N+H O M+S D+C O R$ & 70.2 & 65.0 \\
$M E A N+S D+E N T+C O R$ & 69.6 & 64.2 \\
$M E A N+C O N+E N T+C O R$ & 69.3 & 64.0 \\
$M E A N+H O M+D I S+C O R$ & 69.2 & 63.9 \\
$M E A N+D I S+S D+C O R$ & 69.0 & 63.5 \\
Combinations of five GLCM texture features (top five combinations) & 71.3 & 65.0 \\
$M E A N+S D+E N+A S M+C O R$ & 71.3 & 64.2 \\
$M E A N+H O M+S D+A S M+C O R$ & 70.9 & 64.0 \\
$M E A N+D I S+S D+A S M+C O R$ & 70.8 & 63.9 \\
$M E A N+C O N+E N T+A S M+C O R$ & 70.7 & 63.5 \\
$M E A N+C O N+D I S+A S M+C O R$ & & \\
\hline & & \\
& &
\end{tabular}

number of GLCM texture features, as shown in figure 5. On average, one texture feature achieved $32 \%$ overall accuracy, and this increased to $47 \%$ with combinations of two texture features. Combinations of three texture features were able to produce an average overall accuracy of $63 \%$, rising to $68 \%$ for four texture features and $69 \%$ for five texture features. The overall accuracy increased from one texture feature, to combinations of two, to combinations of three, but levelled off after this point. When four or five texture features were used, the average of overall accuracy from all combinations is close to the overall accuracy produced by the best combination. This indicates that the difference between combinations is small at this level. The standard deviation of overall accuracy for all combinations of four and five texture features is less than $2 \%$. Many combinations of four or five textures can achieve similar results. We can also see from table 1 that the difference in overall accuracy between the top five combinations of five texture features is only around $0.6 \%$. 


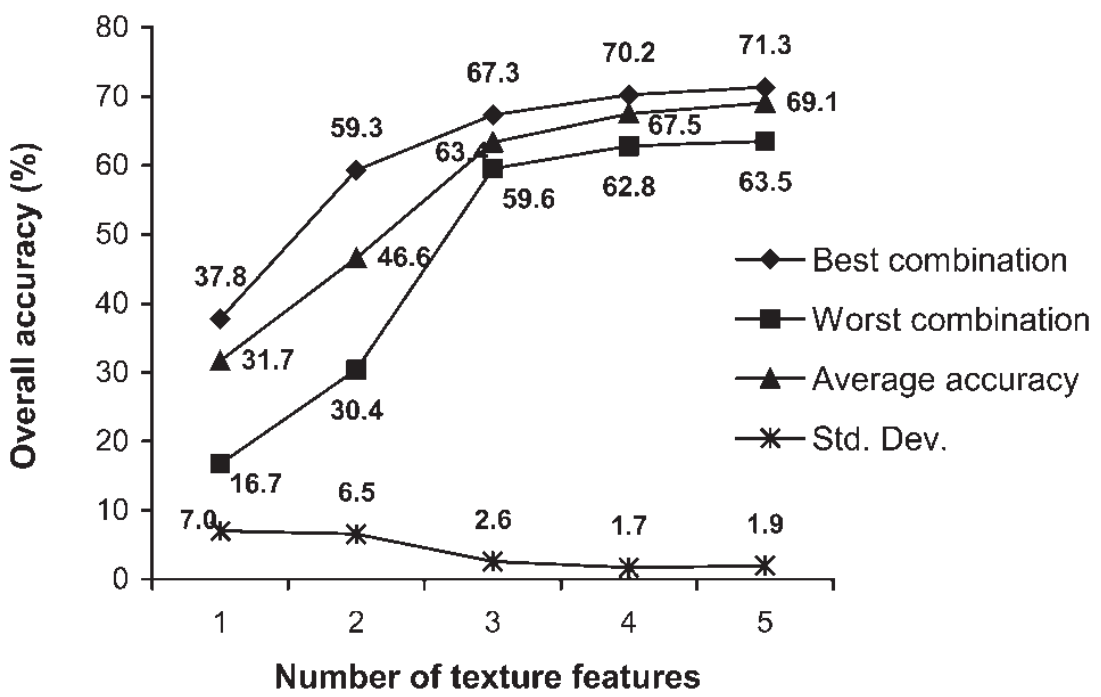

Figure 5. Number of GLCM texture features and classification accuracy for the whole study area.

\subsubsection{The effect of adding other texture features to combinations of GLCM texture features}

The effect of adding ED or NDG to the best combinations of GLCM texture features is shown in table 2. MEAN is combined with NDG or ED for combinations of two instead of DIS (the best single texture feature), because results from the previous section indicated that $M E A N$ combined another GLCM texture produced the best combinations of two texture features. Comparing table 2 and figure 5, we noticed that the combinations involving NDG or ED produced results that had accuracy above or around the average accuracy produced by the same number of GLCM texture features. For example, NDG or ED alone produced an overall accuracy more than the best single GLCM texture feature. $M E A N+\mathrm{NDG}$ or $M E A N+\mathrm{ED}$ produced an overall accuracy that was more than

Table 2. The effect of NDG and ED combined with the best GLCM combinations for the whole study area.

\begin{tabular}{lcc}
\hline Input channels & Overall accuracy $(\%)$ & Kappa $\times 100$ \\
\hline NDG & 42.8 & 31.5 \\
ED & 39.0 & 28.5 \\
$M E A N+\mathrm{NDG}$ & 53.9 & 46.9 \\
$M E A N+\mathrm{ED}$ & 51.6 & 43.5 \\
$M E A N+E N T+\mathrm{NDG}$ & 61.5 & 55.0 \\
$M E A N+E N T+\mathrm{ED}$ & 61.7 & 55.4 \\
$M E A N+E N T+C O R+\mathrm{NDG}$ & 67.9 & 62.3 \\
$M E A N+E N T+C O R+\mathrm{ED}$ & 68.5 & 63.1 \\
$M E A N+H O M+S D+C O R+\mathrm{NDG}$ & 71.9 & 66.9 \\
$M E A N+H O M+S D+C O R+\mathrm{ED}$ & 70.7 & 65.6 \\
$M E A N+S D+E N T+A S M+C O R+\mathrm{NDG}$ & 71.6 & 66.5 \\
$M E A N+S D+E N T+A S M+C O R+\mathrm{ED}$ & 71.8 & 66.9 \\
\hline
\end{tabular}


the average accuracy produced from combinations of two GLCM texture features. Combinations of four or five textures that included NDG or ED also produced an overall accuracy more than the average accuracy from four or five GLCM texture features.

This result suggests that NDG and ED are interchangeable to GLCM texture features, which has a practical application especially when the data-set is big, because it takes a lot of time and computing resources to calculate GLCM texture features. Both NDG and ED are easier to calculate than GLCM texture features.

\subsubsection{Accuracy of individual classes from the best un-stratified classification}

We can see from table 2 that among all the combinations of un-stratified classification, $M E A N+H O M+S D+C O R+$ NDG produced the best result with an overall accuracy of $72 \%$ and a Kappa of 0.67 . Compared this result against reference data, a confusion matrix was produced (table 3 ).

We can see from table 3 that the producer's accuracy is higher than $70 \%$ for five classes out of the eight classes. This is also the case for user's accuracy. The accuracy for 'construction' is the highest and 'old multi-storey' the lowest. Roads are confused with open space or low-rise areas because many roads are covered by trees and the linear shape of roads is not very suitable for texture analysis. Some high-rise areas are confused with low rise and multi-storey areas. High-rise areas are difficult to classify because they do not show the same pattern in the image. There is substantial amount of confusion between 'old multi-storey' and 'new multistorey' or 'low-rise' areas, because texturally, old multi-storey areas may have similar structures as new multi-storey areas and, they may spectrally be close to old low-rise area because the amount of tree coverage. Agriculture areas are confused

Table 3. Confusion matrix for the best un-stratified classification of the study area.

\begin{tabular}{|c|c|c|c|c|c|c|c|c|c|c|}
\hline & & \multicolumn{8}{|c|}{ Reference data } & \multirow{2}{*}{$\begin{array}{c}\text { User's } \\
\text { accuracy }\end{array}$} \\
\hline & & 1 & 2 & 3 & 4 & 5 & 6 & 7 & 8 & \\
\hline \multirow[t]{8}{*}{ Classified data } & 1 & 91.2 & 6.7 & 1.2 & 0.9 & 0.0 & 16.8 & 0.0 & 9.0 & 66.6 \\
\hline & 2 & 0.6 & 72.4 & 14.9 & 2.2 & 17.9 & 19.5 & 0.0 & 4.2 & 78.6 \\
\hline & 3 & 0.0 & 11.3 & 45.9 & 15.2 & 8.5 & 8.6 & 0.0 & 0.4 & 27.8 \\
\hline & 4 & 0.0 & 2.7 & 24.3 & 76.0 & 10.8 & 6.3 & 4.0 & 0.0 & 79.1 \\
\hline & 5 & 0.0 & 0.0 & 0.3 & 4.1 & 58.3 & 0.0 & 0.3 & 0.0 & 84.4 \\
\hline & 6 & 0.4 & 6.5 & 13.5 & 1.6 & 2.3 & 47.3 & 0.0 & 8.5 & 57.3 \\
\hline & 7 & 0.0 & 0.0 & 0.0 & 0.0 & 2.3 & 0.0 & 95.7 & 0.0 & 97.5 \\
\hline & 8 & 7.8 & 0.3 & 0.0 & 0.0 & 0.0 & 1.5 & 0.0 & 78.0 & 91.9 \\
\hline \multicolumn{2}{|c|}{ Producer's accuracy } & 91.2 & 72.4 & 45.9 & 76.0 & 58.3 & 47.3 & 95.7 & 78.0 & 71.8 \\
\hline
\end{tabular}

1. Open space

2. Low-rise

3. Old multi-storey

4. New multi-storey

5. High-rise

6. Roads

7. Construction

8. Agriculture

Note: all numbers are percentages. 
with open space, roads and even low-rise areas. The stratification of the study area would reduce some of the confusion described above.

\subsection{Results from stratified classification}

\subsubsection{Performance of the GLCM texture features in stratified areas}

Table 4 shows classification accuracy for region 1 - the old city, from combinations of different number of GLCM texture features. For region 2-the external city, the results are shown in table 5 and the results for region 3 -non builtup areas are shown in table 6.

Figures 6.1 and 6.2 show that there is similar performance by GLCM texture features in region 1 and region 2 as found for the whole study area. The overall accuracy increases with increasing number of texture features in the combinations. The top combinations of two textures always included $M E A N$ and another texture feature (tables 4 and 5). Two textures are better than one, and three textures are better than two. Four textures are slightly better than three. The combination of

Table 4. Classification accuracy for region 1-old city-using texture features.

\begin{tabular}{|c|c|c|}
\hline Input channels & Overall accuracy (\%) & Kappa $\times 100$ \\
\hline \multicolumn{3}{|l|}{ One GLCM texture feature } \\
\hline$C O N$ & 60.3 & 43.9 \\
\hline$D I S$ & 60.1 & 43.2 \\
\hline HOM & 57.8 & 39.7 \\
\hline ENT & 56.5 & 37.6 \\
\hline$S D$ & 55.8 & 37.7 \\
\hline$A S M$ & 53.2 & 34.2 \\
\hline$M E A N$ & 50.5 & 30.2 \\
\hline COR & 41.3 & 16.6 \\
\hline \multicolumn{3}{|c|}{ Combinations of two GLCM texture features (top five combinations) } \\
\hline$M E A N+E N T$ & 68.3 & 56.9 \\
\hline$M E A N+C O N$ & 68.0 & 56.5 \\
\hline$M E A N+D I S$ & 67.6 & 56.3 \\
\hline$M E A N+H O M$ & 67.6 & 56.3 \\
\hline$M E A N+A S M$ & 66.7 & 55.1 \\
\hline \multicolumn{3}{|c|}{ Combinations of three GLCM texture features (top five combinations) } \\
\hline$M E A N+C O N+E N T$ & 73.8 & 63.9 \\
\hline$M E A N+C O N+C O R$ & 73.7 & 64.1 \\
\hline$M E A N+S D+C O R$ & 73.4 & 63.4 \\
\hline$M E A N+C O N+A S M$ & 73.4 & 63.7 \\
\hline$M E A N+D I S+E N T$ & 73.2 & 63.2 \\
\hline \multicolumn{3}{|c|}{ Combinations of four GLCM texture features (top five combinations) } \\
\hline$M E A N+D I S+E N T+C O R$ & 74.3 & 64.9 \\
\hline$M E A N+C O N+E N T+C O R$ & 74.1 & 64.6 \\
\hline$M E A N+C O N+D I S+C O R$ & 73.9 & 64.4 \\
\hline$M E A N+H O M+C O N+C O R$ & 73.7 & 64.0 \\
\hline$M E A N+H O M+D I S+C O R$ & 73.4 & 63.7 \\
\hline \multicolumn{3}{|c|}{ Combinations of five GLCM texture features (top five combinations) } \\
\hline$M E A N+H O M+C O N+D I S+C O R$ & 74.7 & 65.4 \\
\hline$M E A N+H O M+C O N+D I S+E N T$ & 74.1 & 64.5 \\
\hline$M E A N+C O N+D I S+E N T+C O R$ & 73.9 & 64.5 \\
\hline$M E A N+C O N+E N T+A S M+C O R$ & 73.3 & 63.6 \\
\hline$M E A N+H O M+C O N+E N T+C O R$ & 73.0 & 63.3 \\
\hline
\end{tabular}


four textures $M E A N+D I S+E N T+C O R$ produced the best result for region 1 with $74 \%$ overall accuracy. The best combination of four textures for region 2 is $M E A N+H O M+S D+C O R$, which produced $76 \%$ overall accuracy.

When there are four or five textures in the combinations, the average accuracy they achieved is close to the best combination. Again, this indicates that the difference between one combination and another is small when four or five texture features are used. Any combination of four textures can produce similar result, as can be seen from table 4 and table 5. For region 1, the accuracy of using five textures is actually started to drop from that of four texture features.

Though the trend is similar for the old city and the external city, there is a noticeable difference in the performance. Comparing figures 6.1 and 6.2, we found that the curves for the external city rise more rapidly than those of the old city. One texture achieved an average overall accuracy of 54\% for the old city, while this number is only $45 \%$ for the external city. The average overall accuracy started to decrease when combinations of five texture features are used for the old city, but for the external city, the accuracy increased slightly from combinations of three to four,

Table 5. Classification accuracy for region 2-external city-using texture features.

\begin{tabular}{lcc}
\hline Input channels & Overall accuracy (\%) & Kappa $\times 100$ \\
\hline One GLCM texture feature & & \\
$H O M$ & 56.8 & 39.5 \\
$A S M$ & 55.7 & 36.2 \\
$E N T$ & 55.6 & 37.5 \\
$D I S$ & 53.5 & 35.5 \\
$S D$ & 46.8 & 28.8 \\
$C O N$ & 45.3 & 27.3 \\
$M E A N$ & 33.8 & 22.5 \\
$C O R$ & 10.0 & 0.1 \\
Combinations of two GLCM texture features (top & cive combinations) & \\
$M E A N+H O M$ & 65.7 & 53.9 \\
$M E A N+D I S$ & 62.9 & 50.2 \\
$M E A N+E N T$ & 62.8 & 51.1 \\
$M E A N+A S M$ & 62.0 & 49.0 \\
$M E A N+C O N$ & 61.2 & 48.3 \\
Combinations of three GLCM texture features (top & five combinations) \\
$M E A N+D I S+S D$ & 70.2 & 60.8 \\
$M E A N+H O M+S D$ & 70.1 & 60.9 \\
$M E A N+A S M+C O R$ & 69.1 & 57.9 \\
$M E A N+S D+E N T$ & 68.7 & 59.6 \\
$M E A N+S D+A S M$ & 68.7 & 59.3 \\
Combinations of four GLCM texture features (top five combinations) & \\
$M E A N+H O M+S D+C O R$ & 75.6 & 67.7 \\
$M E A N+S D+A S M+C O R$ & 75.2 & 67.1 \\
$M E A N+S D+E N T+C O R$ & 75.1 & 67.1 \\
$M E A N+D I S+S D+E N T$ & 75.1 & 67.1 \\
$M E A N+C O N+D I S+C O R$ & 74.7 & 66.4 \\
Combinations of five GLCM texture features (top five combinations) & 77.0 & 69.8 \\
$M E A N+H O M+D I S+S D+A S M$ & 76.5 & 69.3 \\
$M E A N+C O N+D I S+S D+A S M$ & 76.4 & 69.1 \\
$M E A N+H O M+C O N+S D+A S M$ & 76.4 & 68.9 \\
$M E A N+H O M+S D+E N T+C O R$ & 76.2 & 68.6 \\
$M E A N+D I S+S D+E N T+C O R$ & & \\
\hline & & \\
& & \\
& &
\end{tabular}


Table 6. Classification accuracy for region 3-non built-up areas-using texture features.

\begin{tabular}{lcc}
\hline Input channels & Overall accuracy $(\%)$ & Kappa $\times 100$ \\
\hline One GLCM texture feature & & \\
$M E A N$ & 91.3 & 79.2 \\
$A S M$ & 59.1 & 15.7 \\
$S D$ & 57.6 & 15.2 \\
$D I S$ & 53.8 & 11.6 \\
$H O M$ & 53.1 & 9.7 \\
$E N T$ & 51.7 & 9.2 \\
$C O N$ & 43.1 & 6.5 \\
$C O R$ & 35.8 & -6.9 \\
Combinations of two GLCM texture features & $($ top five combinations) & \\
$M E A N+S D$ & 92.3 & 81.7 \\
$M E A N+C O R$ & 91.8 & 80.4 \\
$M E A N+H O M$ & 91.5 & 80.0 \\
$M E A N+D I S$ & 91.5 & 80.0 \\
$M E A N+E N T$ & 91.3 & 79.4 \\
Combinations of three GLCM texture features & $($ top five combinations) \\
$M E A N+D I S+C O R$ & 93.7 & 84.7 \\
$M E A N+E N T+C O R$ & 93.3 & 83.9 \\
$M E A N+H O M+C O R$ & 93.3 & 83.8 \\
$M E A N+A S M+C O R$ & 93.3 & 83.6 \\
$M E A N+C O N+D I S$ & 93.1 & 83.2 \\
\hline
\end{tabular}

then from four to five texture features. This may be because the external city is more texturally heterogeneous than the old city. The century-old Chinese style lowrise houses, which appear relatively more homogeneous than multi-storey buildings in the SPOT imagery, are mostly distributed in the old city. There were not many renewal projects underway in the old city around 1994 when the SPOT PAN data were collected. The larger scale new developments were mainly located around the suburban areas.

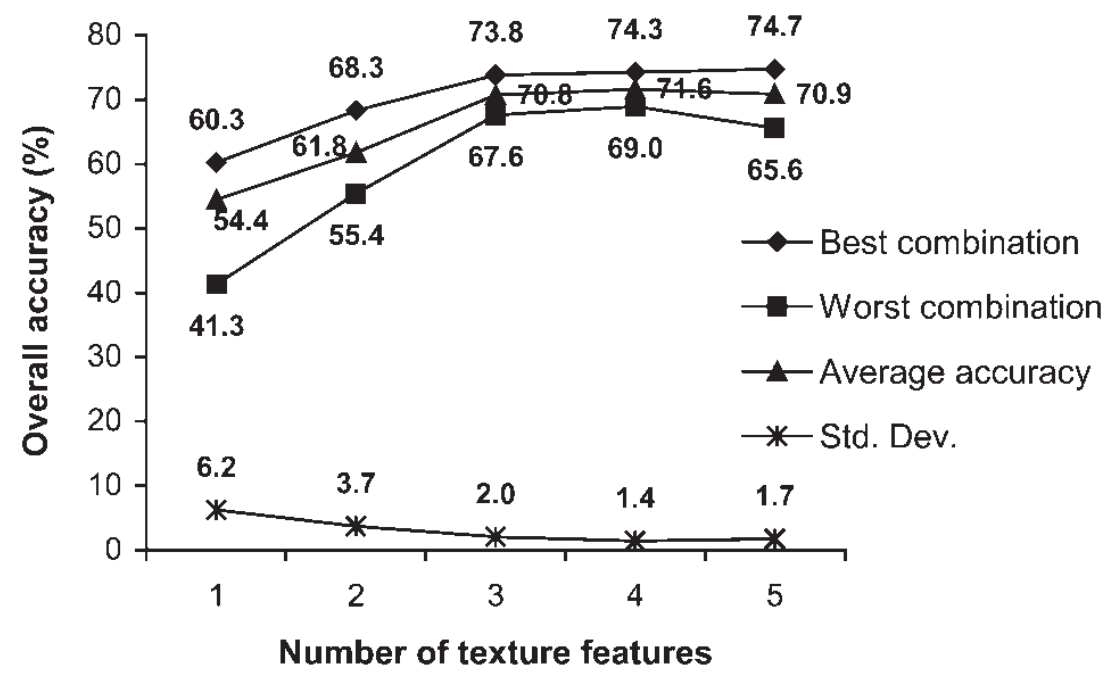

Figure 6.1. Number of GLCM texture features and classification accuracy for the old city. 


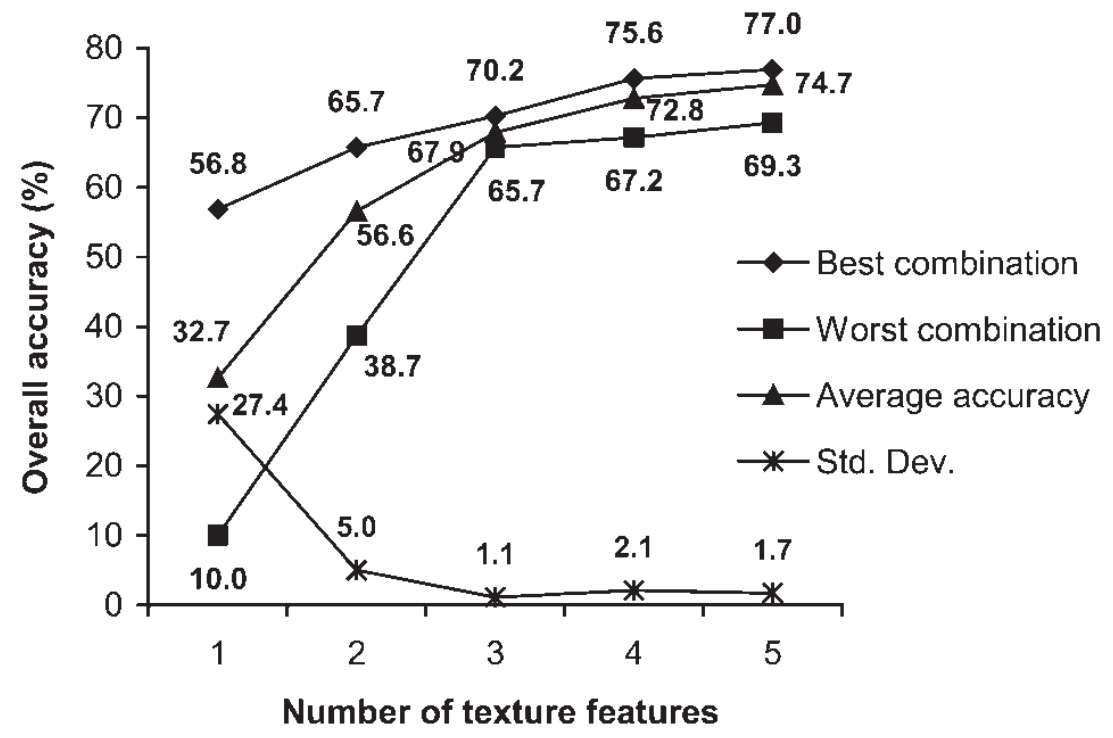

Figure 6.2. Number of GLCM texture features and classification accuracy for the external city.

The texture features perform quite differently for region 3, the non built-up areas (table 6). The MEAN texture alone was able to produce an overall accuracy of $91 \%$ with a Kappa coefficient of 0.79 . Other texture features performed poorly. So that MEAN was kept in the combinations of two and three texture features. There is slightly improvement for two and three texture features, compared with using only MEAN. MEAN $+D I S+C O R$ is the best combination of three texture features, though the difference between the combinations is small-similar to that of the built-up areas. This indicates that in highly homogeneous areas, MEAN is the most effective feature. To classify such homogeneous areas, fewer texture features are needed and spectral information is more important.

\subsubsection{The effect of adding other texture features to combinations of GLCM texture features}

As seen from table 7, the effect of NDG and ED in region 1 and region 2 is similar to the un-stratified classification experiments. Combinations of four or five textures involving NDG and ED can produce an accuracy of approximately the average accuracy produced by the same number of GLCM texture features. This indicates that NDG or ED can be used to replace a GLCM texture feature to reduce computing time.

\subsubsection{Accuracy of the best stratified classification and discussion}

The best classifications for the three stratified regions were combined to produce a classification result for the whole study area. The accuracy of this combined result is shown in table 8. The overall accuracy of the classification is $79 \%$ and the Kappa coefficient is 0.75 . Compared with un-stratified classification, the overall accuracy increased by around $8 \%$ and the Kappa coefficient also increased by $8 \%$. The 
Table 7. The effect of NDG and ED combined with the best GLCM combinations for stratified regions.

\begin{tabular}{lcc}
\hline Input channels & Overall accuracy $(\%)$ & Kappa $\times 100$ \\
\hline Region 1-the old city & & \\
NDG & 57.0 & 38.1 \\
ED & 61.9 & 45.6 \\
$M E A N+\mathrm{NDG}$ & 64.5 & 51.4 \\
$M E A N+\mathrm{ED}$ & 71.2 & 60.5 \\
$M E A N+E N T+\mathrm{NDG}$ & 70.1 & 59.3 \\
$M E A N+E N T+\mathrm{ED}$ & 67.2 & 55.3 \\
$M E A N+C O N+E N T+\mathrm{NDG}$ & 73.4 & 63.5 \\
$M E A N+C O N+E N T+\mathrm{ED}$ & 74.0 & 64.2 \\
$M E A N+D I S+E N T+C O R+\mathrm{NDG}$ & 73.7 & 64.2 \\
$M E A N+D I S+E N T+C O R+\mathrm{ED}$ & 72.5 & 62.5 \\
$M E A N+H O M+C O N+D I S+C O R+\mathrm{NDG}$ & 73.4 & 63.8 \\
$M E A N+H O M+C O N+D I S+C O R+\mathrm{ED}$ & 73.7 & 64.2 \\
Region $2-\mathrm{the}$ external city & & \\
$\mathrm{NDG}$ & 11.6 & 2.0 \\
$\mathrm{ED}$ & 13.5 & 4.7 \\
$M E A N+\mathrm{NDG}$ & 60.0 & 47.8 \\
$M E A N+\mathrm{ED}$ & 59.1 & 46.0 \\
$M E A N+H O M+\mathrm{NDG}$ & 67.8 & 57.2 \\
$M E A N+H O M+\mathrm{ED}$ & 66.1 & 55.0 \\
$M E A N+D I S+S D+\mathrm{NDG}$ & 72.1 & 63.1 \\
$M E A N+D I S+S D+\mathrm{ED}$ & 72.4 & 63.8 \\
$M E A N+H O M+S D+C O R+\mathrm{NDG}$ & 76.0 & 68.3 \\
$M E A N+H O M+S D+C O R+\mathrm{ED}$ & 76.4 & 68.8 \\
$M E A N+H O M+D I S+S D+A S M+\mathrm{NDG}$ & 76.3 & 68.8 \\
$M E A N+H O M+D I S+S D+A S M+\mathrm{ED}$ & 77.3 & 70.3 \\
\hline
\end{tabular}

Table 8. Confusion matrix for the best stratified classification.

\begin{tabular}{lrrrrrrrrrr}
\hline & \multicolumn{8}{c}{ Reference data } & User's \\
\cline { 2 - 8 } & \multicolumn{1}{c}{1} & \multicolumn{1}{c}{2} & \multicolumn{1}{c}{3} & \multicolumn{1}{c}{4} & \multicolumn{1}{c}{5} & \multicolumn{1}{c}{6} & 7 & 8 & accuracy \\
\hline Classified data & 1 & 94.3 & 1.5 & 0.6 & 0.4 & 0.7 & 11.7 & 0.3 & 6.6 & 75.8 \\
& 2 & 0.0 & 77.5 & 13.2 & 0.9 & 0.0 & 7.1 & 0.0 & 1.6 & 87.4 \\
& 3 & 0.0 & 5.8 & 73.1 & 8.0 & 1.6 & 14.6 & 0.0 & 0.0 & 49.2 \\
& 4 & 0.0 & 4.3 & 0.6 & 85.4 & 37.2 & 4.4 & 8.3 & 0.0 & 81.6 \\
& 5 & 0.0 & 0.0 & 0.0 & 2.0 & 59.4 & 0.0 & 0.5 & 0.0 & 89.6 \\
& 6 & 1.6 & 9.9 & 12.6 & 3.1 & 0.0 & 60.5 & 2.7 & 7.8 & 57.7 \\
& 7 & 0.0 & 0.0 & 0.0 & 0.0 & 0.0 & 0.0 & 88.2 & 0.0 & 100 \\
Producer's accuracy & 8 & 4.1 & 1.0 & 0.0 & 0.1 & 1.1 & 1.7 & 0.0 & 84.0 & 93.3 \\
& 94.3 & 77.5 & 73.1 & 85.4 & 59.4 & 60.5 & 88.2 & 84.0 & 79.1 \\
\hline
\end{tabular}

1. Open space

2. Low-rise

3. Old multi-storey

4. New multi-storey

5. High-rise

6. Roads

7. Construction

8. Agriculture

Note: all numbers except class labels are percentages. 
accuracy for six classes out of the total of eight classes is over $70 \%$. The producer's accuracy for five classes (low-rise, old and new multi-storey, roads and agriculture) classes has been increased by over 5\% and over 10\% in particular for low-rise areas and roads, compared with un-stratified classification (table 8 and table 3 ). Though the accuracy for roads and high-rise areas is low, the classification accuracy for other classes is over $73 \%$ (over $84 \%$ for four out of the eight classes). Compared with table 2, the confusion between 'old multi-storey' and 'new multi-storey', between 'low-rise' and 'old multi-storey', and between 'agriculture' and 'open space' is greatly reduced, because of the different classification schemes used for the stratified regions.

The stratified classification result shows clearly the spatial pattern of the city in 1994 (figure 7). The old city was made up of mostly low-rise and old multi-storey buildings, along with a few big open water areas and vegetated areas. The external city is a mosaic of multi-storey areas of all periods. High-rise buildings were dotted around the band between the Second Ring Road and the Third Ring Road and some in the most recently built areas beyond the Third Ring Road. Green areas show the parks and waterways. Big construction sites are at the edge of the city. Some agricultural fields were distributed in the urban-rural fringe.

We can see from figure 7 that the study area is fragmented. This may be explained by: (1) the border problem of texture analysis; and (2) the fragmentation of the city in reality. Texture analysis involves a window of a defined size. In the boundary zone of two or more classes, texture features calculated from the window are influenced by more than one class. Further classification of the texture features is likely to produce a border between two classes. This imposes a problem when every pixel in a scene is classified. The larger the window size used to produce the texture features, the more evident the border problem. Though many studies have found a larger window size resulted in higher classification accuracy (Karathanassi et al. 2000, Pesaresi 2000), an appropriate window size is needed to achieve the desirable accuracy and reduce the border problem. We considered the size of the feature to be identified and the spatial resolution of the imagery when selecting the window size. In this study, the GLCM texture features were produced from a $7 \times 7$ window on the SPOT PAN image, which is able to capture the textural characteristics of various urban spatial patterns in the study area. This window size is relatively small compared with some of the recommended window sizes in the literature. For example, Karathanassi et al. (2000) suggested a window size of $31 \times 31$ pixels or larger for classification of urban area into low, medium and high density regions using texture features from SPOT panchromatic data. The border problem is not quite as serious at a window size of $7 \times 7$ pixels, though we do see some of the border effects in figure 7.

In addition to the border problem, the urban landscape of the city of Beijing is fragmented in reality. Though the development in the last 20 years resulted in a modern city, the landscape of Beijing is different from a North American city. We see clearly commercial, industrial, multi-family and single family residential areas with definitely different patterns in a North American city. However, there are lowrise, multi-storey and high-rise areas interweaving with each other in Beijing. In the old city, century-old residential areas are next to 3-5 storey government buildings and industrial-residential compounds built in the 1950s and 1960s. In the external city, compounds built in the 1950 s and 1960 s were next to new multi-storey 
buildings built in the 1980s and 1990s. High-rise buildings were built along major roads, usually with multi-storey buildings behind. The classification results differentiate 'old multi-storey' (compounds) from 'new multi-storey' (1980s and later), which indicate different living conditions in the multi-storey buildings. New multi-storey residential buildings usually contain complete units of apartments, but people living in the 'old multi-storey' buildings may have to use shared kitchens and other spaces. Low-rise buildings outside of the old city include some earlier residential areas near the old city wall, previous rural settlements in transition and some temporary structures. This mosaic of various urban built-up patterns

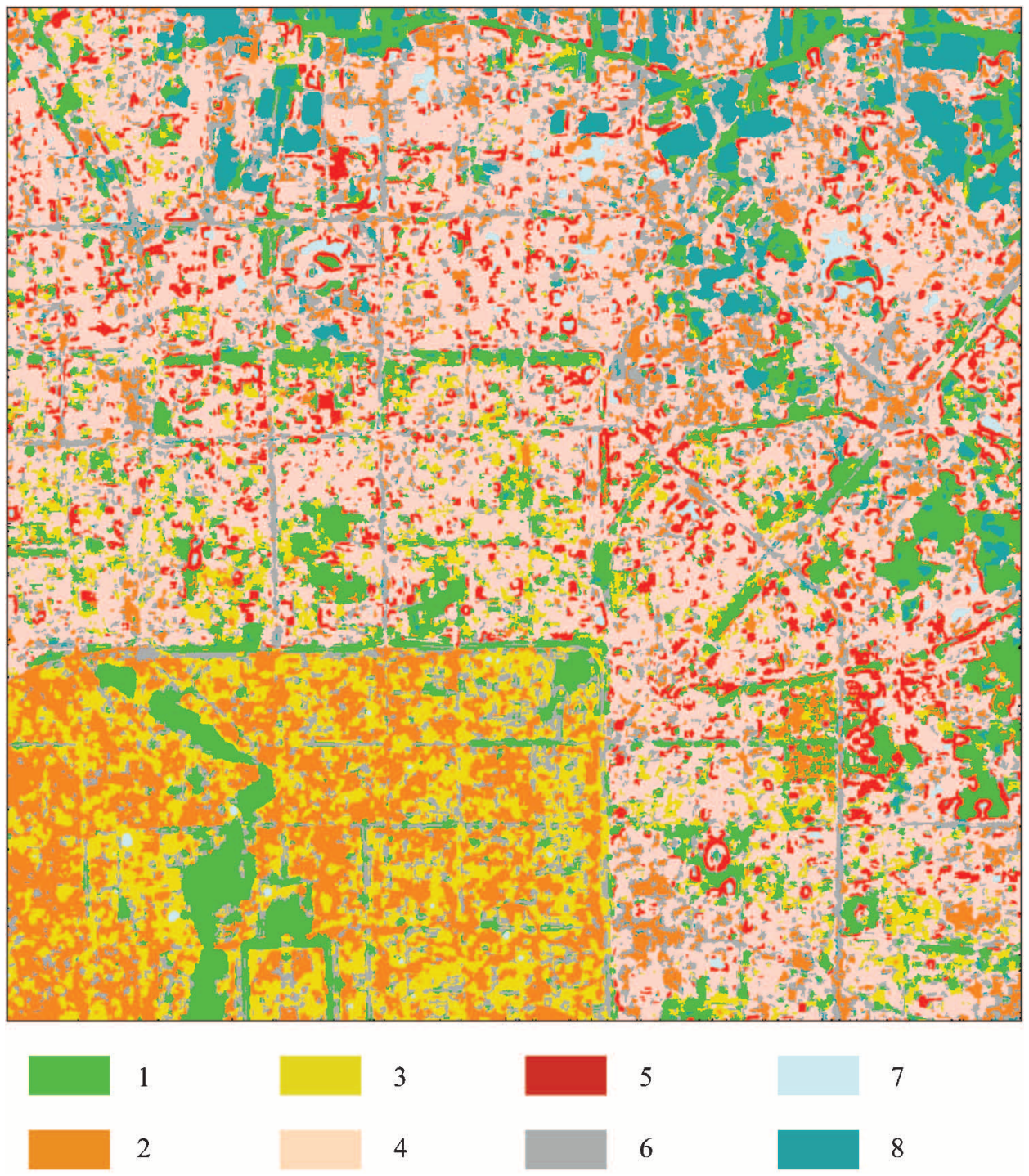

Figure 7. Classification result from stratified classification. (1) Water/tree/grass, (2) low-rise, (3) old multi-storey, (4) new multi-storey, (5) high-rise, (6) roads, (7) construction, and (8) agriculture. 
developed from different time periods is unique in the case of Beijing and the spatial patterns change over time. Figure 7 shows the general distribution of these different areas in 1994, which is useful information for urban landscape study and future urban development decisions.

\subsection{General discussion on the selection of GLCM texture features}

The performance of the eight GLCM texture features is evaluated in this study. Since some of the texture features are highly correlated, usually we do not need all of them to perform the classification. The question is, then, how do we select from the eight? Though this study does not focus on which texture features are better than others, there are some guidelines. Haralick et al. (1973) demonstrated the usefulness of $A S M, C O N, C O R$ and ENT among the set of texture features they proposed. In the study by Gong et al. (1992), three GLCM texture features including ASM, CON and COR were found to be less correlated. Hall-Beyer (2000) divided the eight GLCM texture features used in this study into three groups: the 'contrast' group (CON, DIS and HOM), the 'orderliness' group (ASM and ENT) and the 'descriptive statistics' group (MEAN, $S D$ and $C O R)$. The texture features in the contrast group are correlated with each other, so are the features in the orderliness group. $M E A N$ and $C O R$ are generally not correlated with other features. She suggested that researchers choose one measure from the contrast group, one from the orderliness group and two or three from the descriptive statistics group for classification purposes. This would result in a combination of four or five texture features that capture different aspects of the GLCM. The findings of the present study are consistent with these previously reported results. Careful examination of the best combinations of three, four or five GLCM texture features in tables 1, 4, 5, 6 shows that the texture features in the best combinations tend to be from these different groups. Figures 5, 6.1 and 6.2 also show that when we use four or five texture features, the difference between different combinations actually becomes smaller.

\section{Conclusions}

Different texture features, including eight texture features from the GLCM method, the computationally-simple NDG texture, and the structural texture ED were evaluated for urban spatial pattern classification of Beijing, China, from $10 \mathrm{~m}$ spatial resolution SPOT PAN imagery. The texture features were used as input channels to the supervised classifier. The classifications were performed in the study area as a whole (un-stratified) and separately in three texturally different regions: the old city, the external city and non built-up areas (stratified). The results show that visually different urban spatial patterns on the SPOT panchromatic imagery could be classified using texture features. The performance of texture features in this study can be summarized as follows:

1. Results from single texture feature were generally poor except that $M E A N$ alone was able to produce over $90 \%$ accuracy in texturally homogeneous non built-up areas.

2. Classification accuracy increased with increasing number of GLCM texture features up to combinations of three or four texture features, where the accuracy levelled off. 
3. When four or more GLCM texture features were combined, many different combinations performed about as well.

4. MEAN combined with another GLCM texture feature produced the best results among all combinations of two GLCM texture features.

5. DNG and ED can be used to substitute some GLCM texture features to reduce computing time.

6. Texture features performed differently in texturally different regions in the study area. For more homogeneous regions, single or combinations of two texture features had better performance, and fewer numbers of texture features were needed to approach the peak of classification accuracy.

The best un-stratified classification in this study produced an overall accuracy of $72 \%$ for the whole study area, with a Kappa coefficient of 0.67 . This is a large improvement over the accuracy of original SPOT PAN imagery alone $(27 \%)$. The best stratified classification, which is the combination of the best classifications from the three stratified regions, was able to achieve an overall accuracy of $79 \%$ and a Kappa coefficient of 0.75 , which is about two times better than the result from original SPOT PAN imagery. This shows clearly the spatial pattern of the city.

\section{Acknowledgements}

This research was partly funded by a Natural Science and Engineering Council (NSERC) grant awarded to Dr Wang and grants from the National Natural Science Foundation of China awarded to Dr Gong and Dr Shi. This research was supported by the Visiting Scholar Foundation of the Key Laboratory of Environmental change and Natural Disaster, the Ministry of Education of China, Beijing Normal University.

\section{References}

ARZANDEH, S., 2001, Evaluation of radar and optical satellite imagery for wetland mapping and change detection in Walpole Island, Ontario. Unpublished Masters thesis. Department of Geography, The University of Western Ontario, Canada.

ARZANDEH, S., and WANG, J., 2002, Texture evaluation of Radarsat imagery for wetland mapping. Canadian Journal of Remote Sensing, 28, 653-666.

BARAldi, A., and PARMiggiani, F., 1995, An investigation of the textural characteristics associated with gray level cooccurrence matrix statistical parameters. IEEE Transactions on Geoscience and Remote Sensing, 33, 293-304.

BARNSLEY, M., and BARR, S., 1996, Inferring urban land use from satellite sensor images using kernel-based spatial reclassification. Photogrammetric Engineering and Remote Sensing, 62, 949-958.

ConNers, R. W., and Harlow, C. A., 1980, A theoretical comparison of texture algorithms. IEEE Transactions On Pattern Analysis and Machine Intelligence, PAMI-2, 204-222.

FORSTER, B., 1985, An examination of some problems and solutions in monitoring urban areas from satellite platforms. International Journal of Remote Sensing, 6, 139-151.

Gaubatz, P., 1995, Changing Beijing. The Geographical Review, 85, 79-96.

GONG, P., and HowARTH, P. J., 1990, The use of structural information for improving landcover classification accuracies at the rural-urban fringe. Photogrametric Engineering and Remote Sensing, 56, 67-73.

Gong, P., Marceau, D. J., and Howarth, P. J., 1992, A comparison of spatial feature extraction algorithms for land-use classification with SPOT HRV data. Remote Sensing of Environment, 40, 137-151.

HAACK, B., BRYANT, N., and ADAMS, S., 1987, An assessment of Landsat MSS and TM data for urban and near-urban land-cover digital classification. Remote Sensing of Environment, 21, 201-213. 
HALl-BeYer, M., 2000, GLCM texture: a tutorial. Available on line at http://www.ucalgary. $\mathrm{ca} / \sim$ mhallbey/texture/texture_tutorial.html (22 July 2002).

Haralick, R. M., 1979, Statistical and structural approaches to texture. Proceedings of the IEEE, 67, 786-804.

Haralick, R. M., Shanmugam, K., and Dinstein, I., 1973, Textural features for image classification. IEEE Transactions on System, Man, and Cybernetics, SMC-3, 610-621.

HARRIS, P. M., and VenturA, S. J., 1995, The integration of geographic data with remotely sensed imagery to improve classification in an urban area. Photogrametric Engineering and Remote Sensing, 61, 993-998.

Hornstra, T. J., Lemmens, M. J. P. M., and Wright, G. L., 1999, Incorporating intra-pixel reflectance variability in the multispectral classification process of high-resolution satellite imagery of urbanised areas. Cartography, 28, 1-9.

Karathanassi, V., Iossifidis, CH., and RokOS, D., 2000, A texture-based classification method for classifying built areas according to their density. International Journal of Remote Sensing, 21, 1807-1823.

Marceau, D. J., Howarth, P. J., Dubois, J. M., and Gratton, D. J., 1990, Evaluation of the grey-level co-occurrence matrix method for land-cover classification using SPOT imagery. IEEE Transactions on Geoscience and Remote Sensing, 28, 513-519.

Mesev, V., 1998, The use of census data in urban image classification. Photogrammetric Engineering and Remote Sensing, 64, 431-438.

PeSARESI, M., 2000, Texture analysis for urban pattern recognition using fine-resolution panchromatic satellite imagery. Geographical and Environmental Modelling, 4, 43-64.

ShabAN, M. A., and DiKshit, O., 2001, Improvement of classification in urban areas by the use of textural features: the case study of Lucknow city, Uttar Pradesh. International Journal of Remote Sensing, 22, 565-593.

WANG, F., and ZHOU, Y., 1999, Modelling urban population densities in Beijing 1982-90: suburbanisation and its causes. Urban studies, 36, 271-287.

WANG, J., 1995, Application of the Linear Feature Detection System-LINDA to image segmentation from remotely sensed data. Remote Sensing Reviews, 13, 49-66.

WANG, J., and HowARTh, P. J., 1991, Structural measures for linear feature pattern recognition from satellite imagery. Canadian Journal of Remote Sensing, 17, 294-303.

Webster, C. J., 1996, Population and dwelling unit estimates from space. Third World Planning Review, 18, 155-176.

WelCH, R., 1982, Spatial resolution requirements for urban studies. International Journal of Remote Sensing, 3, 139-146.

Weszka, J. S., Dyer, C. R., and Rosenfeld, A., 1976, A comparative study of texture measures for terrain classification. IEEE Transactions on System, Man, and Cybernetics, SMC-6, 269-285.

WU, L., 1986, A brief history of ancient Chinese city planning: in urbs et regio, (Kassel: Gesamthochschulbibliothek).

WU, L., 1999, Rehabilitating the old city of Beijing: a project in the Ju'er Hutong neighbourhood. (Vancouver: UBC Press).

ZHANG, Q., and WANG, J., 2003, A rule-based urban land use inferring method for fine resolution multispectral imagery. Canadian Journal of Remote Sensing, (in press).

Zhang, Q., Wang, J., Peng, X., Gong, P., and Shi, P., 2002, Urban built-up land change detection with road density and spectral information from multi-temporal Landsat TM data. International Journal of Remote Sensing, 23, 3057-3078. 